Check for updates

Cite this: RSC Adv., 2017, 7, 33961

\title{
Step-feed strategy enhances performance of unbuffered air-cathode microbial fuel cells $\dagger$
}

\author{
Liang Zhang, (D)*abc Xun Zhu, ${ }^{\text {ab }}$ Jun Li, (D) ab Hiroyuki Kashima, ${ }^{c}$ Qiang Liao ${ }^{\text {ab }}$ \\ and John M. Regan ${ }^{c}$
}

Step-feed anolyte recirculation is compared with single-inlet recirculation feed in a single-chamber aircathode microbial fuel cell (MFC) under buffer-less conditions, to examine the effects of distributing the organic load and cathodically increased electrolyte $\mathrm{pH}$ across the anode. The step-feed effects on proton transfer, maximum power output, and chemical oxygen demand removal are investigated. The results show that better proton transfer could be induced by using a step-feed configuration with uniform flow distribution across four anode sections of MFCs. Thus, the maximal power density is increased from $3.5 \mathrm{~W} \mathrm{~m}^{-3}$ to $4.12 \mathrm{~W} \mathrm{~m}^{-3}$, indicating a $17.7 \%$ increase in the maximum power density. A slight increase $(9.9 \%)$ is obtained in coulombic efficiency (CE, $11.1 \%$ ). Additional $5.1 \%$ increases in power $\left(4.33 \mathrm{~W} \mathrm{~m}^{-3}\right.$ ) and $15 \%$ increases in CE (approximately 12.5\%) are observed after increasing the flow distribution toward the anode section closest to the cathode. This study demonstrates that step-feed could be a beneficial operational strategy for future MFC designs.

Received 1st April 2017

Accepted 30th June 2017

DOI: $10.1039 / c 7 r a 03769 d$

rsc.li/rsc-advances oxygen transport to the anode, yielding an optimal recirculation flow rate that considered the competing factors of proton and oxygen transfer. ${ }^{\mathbf{1 4}}$ It was reported that the benefits from the anolyte recirculation strategy were to avoid the risk of eutrophication in downstream aquatic systems due to phosphorus discharge, to eliminate the expense of adding phosphate and to obtain a higher Coulombic efficiency of MFCs. ${ }^{14}$ It should be noted that the anolyte recirculation also had several operational disadvantages such as addition energy required for pumping, longer treatment times, and less power output. ${ }^{14}$ However, it was obvious that the advantages of the anolyte recirculation strategy outweighed its drawbacks due to cost and environmental considerations. These previous studies indicated that anolyte recirculation could be a viable strategy to enhance proton transport for the application of MFCs under buffer-less conditions.

Scale-up is another important consideration and bottleneck in the application of MFC technologies. ${ }^{20}$ Limitations such as uneven mass distribution, poor substrate utilization, low power output, and non-uniform current distribution will be present in large-scale continuous-flow MFCs. ${ }^{21,22}$ Similar problems occur in other bioprocesses, and step-feed strategies have been adopted to support more homogeneous mass distribution and improve reactor performance. ${ }^{23-25}$ In our previous study of a single-chamber system, higher $\mathrm{pH}$ electrolyte near the air cathode was recirculated to the distal end of the brush anode. While this improved performance relative to a control without recirculation, it could not fully compensate for acid production across the entire anode, ${ }^{\mathbf{1 4}}$ so it improved the $\mathrm{pH}$ biocompatibility primarily in the anode section that might contribute the

\footnotetext{
${ }^{a}$ Key Laboratory of Low-grade Energy Utilization Technologies and Systems (Chongqing University), Ministry of Education, Chongqing 400030, China. E-mail: liangzhang@ cqu.edu.cn; Fax: +86-23-6510-2474; Tel: +86-23-6510-3102

${ }^{b}$ Institute of Engineering Thermophysics, Chongqing University, Chongqing 400030, China

${ }^{c}$ Department of Civil and Environmental Engineering, The Pennsylvania State University, University Park, PA 16802, USA

$\dagger$ Electronic supplementary information (ESI) available. See DOI: 10.1039/c7ra03769d
} 
least to current production. ${ }^{22}$ Step-feed recirculation across the anode volume might further increase the benefits of recirculation by improving the $\mathrm{pH}$ in multiple anode sections, including more bioelectrochemically active anode volumes.

In this study, the application of step-feed in anolyte recirculation was investigated for the batch-fed operation of unbuffered single-chamber air-cathode MFCs. The step-feed effects on proton transfer and system performance were measured. Considering the nonuniform anodic current distribution on large electrodes, ${ }^{22}$ uneven flow distribution of stepfeed operation toward the cathode was also investigated.

\section{Materials and methods}

\subsection{MFC construction and operation}

A single-chamber air-cathode MFC was constructed by connecting a vertical Plexiglass cylinder anode chamber (length $=$ $12 \mathrm{~cm}$, inner diameter $=6.35 \mathrm{~cm}$, liquid volume $=340 \mathrm{~mL}$ ) and a rectangular Plexiglass cathode chamber $(15 \mathrm{~cm} \times 8 \mathrm{~cm})$ with a $6.35 \mathrm{~cm}$ diameter hole (Fig. 1). A large carbon brush (length $=$ $7 \mathrm{~cm}$, diameter $=6 \mathrm{~cm}$, PANEX $33160 \mathrm{~K}$, ZOLTEK) was vertically arranged in the middle of the cylindrical chamber. A floating cathode was constructed of wet-proofed (30\%) carbon cloth (14 $\mathrm{cm} \times 8 \mathrm{~cm}$, type A, E-TEK) coated with a carbon base layer on the air side and a $0.5 \mathrm{mg}-\mathrm{Pt} \mathrm{cm}^{-2}$ catalyst layer on the liquid side. A rectangular hole $(2 \mathrm{~cm} \times 1 \mathrm{~cm})$ in the carbon cloth over the middle of the cylindrical chamber was used to pass the titanium wire core of the carbon-brush anode and to collect $\mathrm{pH}$ and COD samples. A nonconductive tube was attached around the wire core to avoid a short circuit. The reactors were operated in a loop mode. The cathode effluent overflowed into a $100 \mathrm{~mL}$

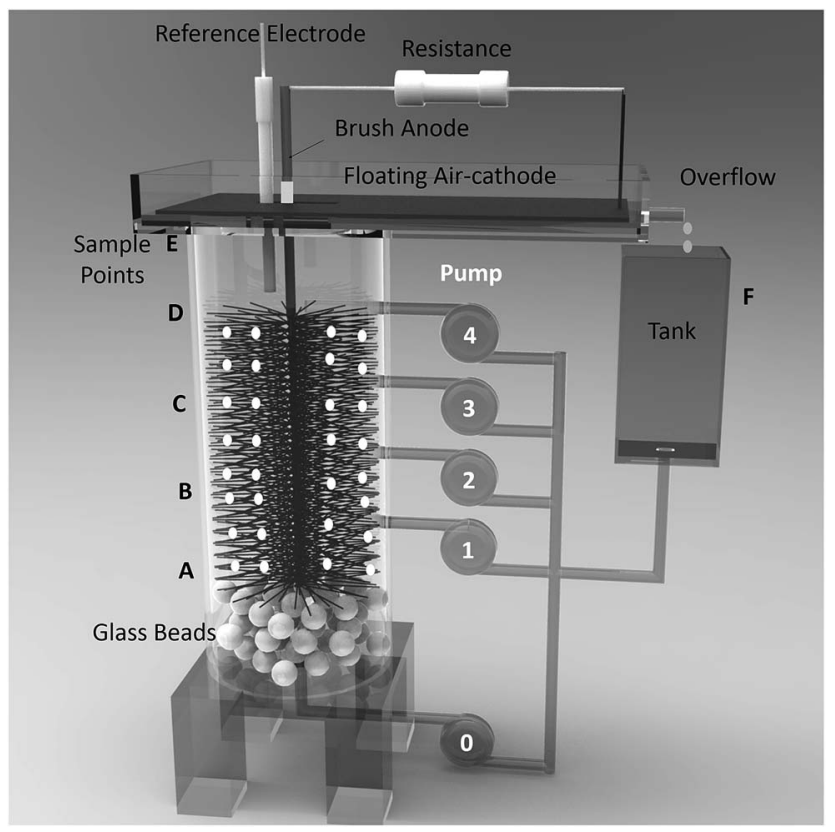

Fig. 1 Schematic of MFC reactor with step-feed in anolyte recirculation. Numbers 0 through 4 indicate feeding inlets, and letters $A$ through $\mathrm{F}$ indicated sampling locations. volumetric flask (Tank) and then was pumped into the bottom of the anode. A packed bed of $1.0 \mathrm{~mm}$ diameter glass beads (height $=3 \mathrm{~cm}$ ) was installed at the inlet of the anode to ensure a uniform flow of the influent electrolyte.

To investigate the step-feed recirculation strategy in MFC operation, four inlets evenly distributed along the vertical anode chamber were added into the middle of the reactor (notation for inlets: 0 - bottom of reactor, $1-1 / 4$ of brush height, $2-1 / 2$ of brush height, $3-3 / 4$ of brush height, and $4-$ top of brush). The flow rate of each inlet was individually controlled by a peristaltic pump. An $\mathrm{Ag} / \mathrm{AgCl}$ reference electrode (RE-5B, BASi, $+0.21 \mathrm{~V}$ versus standard hydrogen electrode) was placed in the top of the anode chamber.

The MFC was inoculated in the first batch with a $50: 50$ mixture of unbuffered medium $\left(1.28 \mathrm{~g} \mathrm{~L}^{-1} \mathrm{CH}_{3} \mathrm{COONa}, 3.38 \mathrm{~g}\right.$ $\mathrm{L}^{-1} \mathrm{KCl}, 0.07 \mathrm{~g} \mathrm{~L}^{-1} \mathrm{NaH}_{2} \mathrm{PO}_{4} \cdot \mathrm{H}_{2} \mathrm{O}, 0.31 \mathrm{~g} \mathrm{~L}^{-1} \mathrm{NH}_{4} \mathrm{Cl}$, and trace minerals and vitamins; initial $\mathrm{pH}$ of 7.04 , conductivity of 7.60 $\mathrm{mS} \mathrm{cm}{ }^{-1}$ ) and domestic wastewater from the primary clarifier of the Pennsylvania State University Wastewater Treatment Plant. After the voltage decreased to less than $5 \mathrm{mV}$ in the first batch, sterile unbuffered medium was used to refill the reactor for later batches. During the startup period, the MFC was first acclimated to a high external resistance of $1000 \Omega$ to obtain a steady current production in several batches, and then to lower resistances of 100 and $50 \Omega$ to produce higher current. In the batch experiments, the external resistance of the MFC was set at $50 \Omega$. The reactor was operated in a water bath with a stable temperature of $25{ }^{\circ} \mathrm{C}$. The $\mathrm{MFC}$ was operated under four sequential cases (Table 1), with two batches per condition: Case 1 was a single-inlet recirculation feed at the bottom of the reactor, Cases 2 and 3 had uniform step-feed flow distributions, and Case 4 had a higher flow in the terminal section of the anode closest to the cathode electrode.

\subsection{Measurements and calculations}

Cell voltages and the anode and cathode potentials were collected every $30 \mathrm{~s}$ using a data acquisition unit (Model 2700, Keithley Instruments) connected to a PC. Current density and power density were calculated as previously described ${ }^{\mathbf{1}}$ based on the anode liquid volume (for $\mathrm{W} \mathrm{m}^{-3}$ ). Before the polarization test at each case, the MFC was operated at $50 \Omega$ for $12 \mathrm{~h}$ after replacing with fresh medium and then operated under opencircuit condition for over $3 \mathrm{~h}$. During the polarization process, the external resistance was varied over the range of $1.0 \times 10^{5}$ to $5 \Omega$ and voltage was recorded after $30 \mathrm{~min}$ of operation at each

Table 1 Recirculation flow rates of MFC operated under different cases

\begin{tabular}{llllll}
\hline & \multicolumn{5}{l}{ Flow rate $\left(\mathrm{mL} \mathrm{min}^{-1}\right)$} \\
\cline { 2 - 6 } $\begin{array}{l}\text { Operational } \\
\text { strategy }\end{array}$ & 0 & 1 & 2 & 3 & 4 \\
\hline Case 1 & 0.4 & 0 & 0 & 0 & 0 \\
Case 2 & 0.1 & 0.1 & 0.1 & 0.1 & 0 \\
Case 3 & 0 & 0.1 & 0.1 & 0.1 & 0.1 \\
Case 4 & 0 & 0.1 & 0.1 & 0.2 & 0
\end{tabular}


external resistance. The $\mathrm{pH}$ distribution throughout the system and COD in the anode and recirculation tank were monitored during the batch experiments. Samples for $\mathrm{pH}$ tests were collected from six sample points (A - bottom brush, B - 1/3 of brush height, $\mathrm{C}-2 / 3$ of brush height, D - top of brush, E adjacent to the surface of the cathode, $\mathrm{F}$ - recirculation tank). COD of anode chamber (sample point in the middle of B and C) and tank was measured in duplicate using a low range (0$150 \mathrm{mg} \mathrm{L}^{-1}$ ) HACH COD kit (Hach Co., Loveland, CO) and $\mathrm{pH}$ was measured using a Multiparameter meter (AG, MettlerToledo). The interval of collecting COD samples were approximately 3 hours for the first 12 hours of each batch and 10-20 hours for the rest of batch. The coulombic efficiency (CE) was calculated based on COD removed, as previously described. ${ }^{1}$

\section{Results and discussion}

\subsection{Effect of step-feed on electricity generation}

Repeatable and different voltage outputs across $50 \Omega$ were observed for each operational strategy of anolyte recirculation (Fig. 2a). Case 1, which served as the benchmark condition with recirculation only from the bottom inlet, had a plateau voltage of approximately $0.23 \mathrm{~V}$ and an average batch time of $80 \mathrm{~h}$. A significant increase (average 8.7\%) was observed in the voltage output after switching to step-feed operation (Case 2), and Cases 3 and 4 had approximately the same plateau voltage output as Case 2 but longer batch times. Inspection of individual electrode potentials showed that all three step-feed configurations resulted in increased cathode potentials compared with Case 1, which were partially offset by slightly higher anode potentials (Fig. 2b).

The changes in electrode potentials might be attributed to the different electrolyte $\mathrm{pH}$ profiles under different operational cases, as well as the effect of recirculating oxygen (which was not measured) only to the bottom of the anode versus distributing it across the anode volume. Uneven $\mathrm{pH}$ distributions were found during batch operation in all cases (Fig. S1 $\dagger$ ). The anodic pHs (from sample point A to E) showed a quick drop in the first $10 \mathrm{~h}$ of a batch and then slightly increased over the rest of batch operation. No appreciable $\mathrm{pH}$ change was observed in the recirculation tank after the cathode, except at the beginning and ending of each batch. The baseline operating condition (Case 1) generally had a slightly higher anodic $\mathrm{pH}$ distribution, which would be less inhibitory to microbial activity and likely contributed to the lower anode potential observed in this condition (Fig. 2b). It should be noted that the recirculation inlets and the $\mathrm{pH}$ measurement locations were offset by approximately $1.0 \mathrm{~cm}$, so there may have been increases in $\mathrm{pH}$ at the inlets that were not captured in the $\mathrm{pH}$ profiles. The lower pHs adjacent to the surface of the cathode in all three step-feed configurations (Fig. 2c) significantly improved the cathode performance compared with Case 1 and offset the less negative anode potential differences.

Nonuniform anodic current distribution was reported in a liter-scale MFC with a large anode electrode, with the anode section closest to the cathode electrode generating higher current density. ${ }^{22}$ Therefore, it was expected that a higher
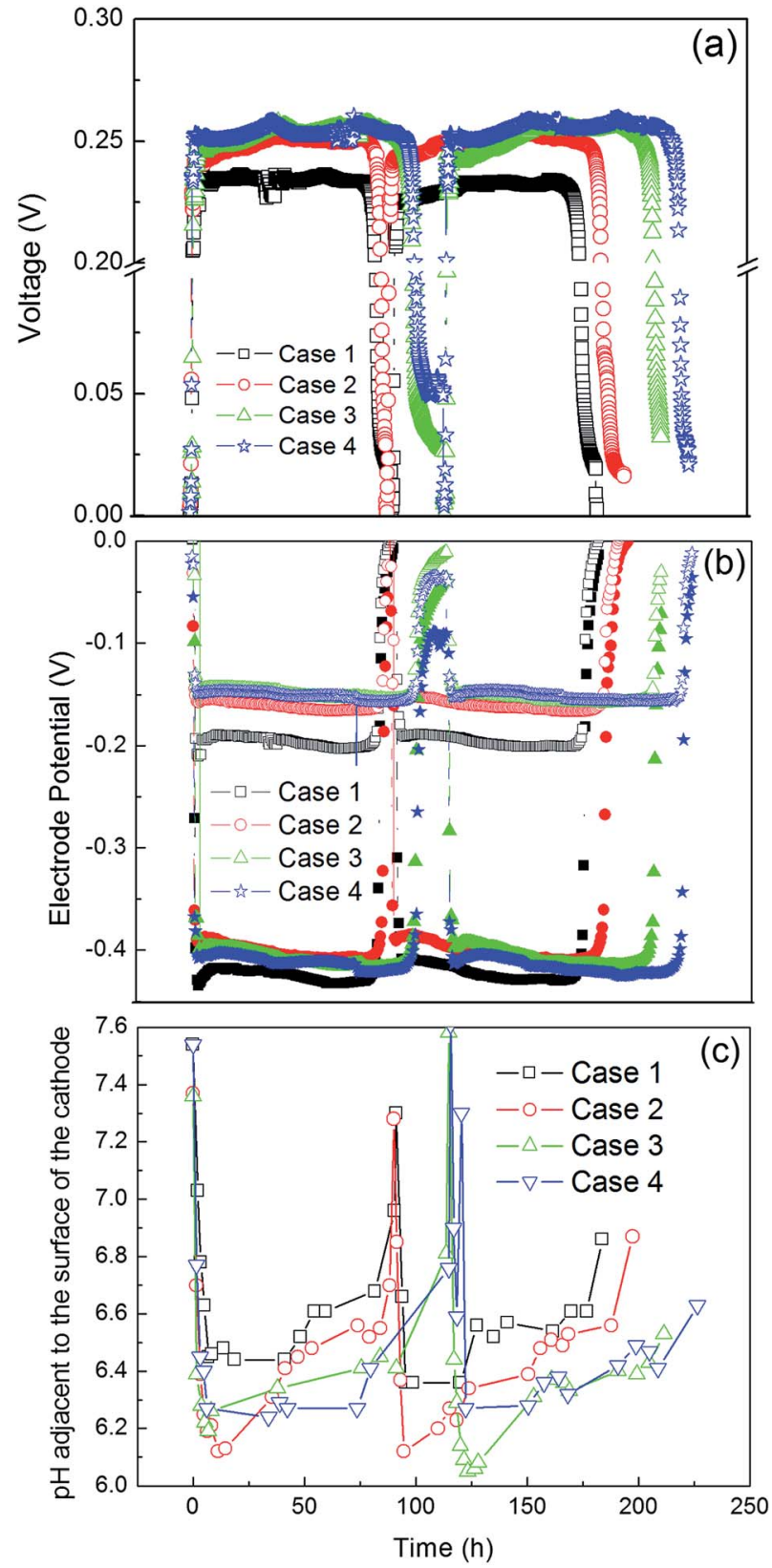

Fig. 2 Voltage output (a), electrode potentials (b) and $\mathrm{pH}$ adjacent to the surface of the cathode (c) in batch operation of unbuffered MFC under different cases (in (b), open and closed symbols indicate the cathode potential and the anode potential, respectively).

voltage output could be obtained by increasing the recirculation flow distribution of the anode closer to the cathode electrode. This expectation was supported by the slight increase in cell voltage and longer operating times of Cases 3 and 4 relative to the uniform distribution of Case 2.

\subsection{Effect of step feed on COD removal and CE}

COD in the middle of sample point $\mathrm{B}$ and $\mathrm{C}$ and in the recirculation tank was measured during the batch operation under each condition (Fig. S2 $\dagger$ ). The CODs dropped rapidly in the first 
several hours and then gradually decreased until the batches ended. This was reflected in the unbuffered anolyte $\mathrm{pH}$ response as well, with an initial decrease down to as low as 6.0 and then a gradual increase (Fig. S1 $\dagger$ ). At the end of each batch, the averaged $\mathrm{pH}$ of electrolyte was nearly neutral (Fig. S3†). As shown in Fig. S3, $\uparrow$ the final low COD resulted in the batch ending (with respect to electricity generation) for unbuffered MFCs, similarly with previous buffered MFCs. ${ }^{14}$ In addition, step-feed operation led to lower final COD with longer batch time, resulting in a slightly higher COD removal percentage (Fig. 3). This was probably the fact that step-feed induced an uneven mass distribution and then a high substrate utilization and a high current generation. This was consistent with previous reports of other bioprocesses, in which step-feed operation improved substrate removal in wastewater. $^{24,26}$ While the CEs were relatively low in this MFC design, a slight increase was observed after using step-feed operation. Compared with the CE of Case 1 (10.1\%), the MFC operated with uniform step feed (Case 2) had a CE of $11.1 \%$. The increase in $\mathrm{CE}$ was attributed that step-feed resulted in the enhancement of proton transfer, providing a favorable microenvironments for the growth of electrochemically active bacteria and a relatively low $\mathrm{pH}$ environment for cathode reaction. As a result, a high current generation was obtained in MFCs. Meanwhile, it was reported that the increasing recirculation rate brought more oxygen into an anode and caused a lower CE in MFCs with anolyte recirculation. ${ }^{\mathbf{1 4}}$ It should be noted that the total flow rate of step-feed was maintained unchanged, suggesting the similar amount of oxygen transfer into the anode. Thus, the enhanced proton transfer from the step-feed operation mainly attributed the higher CE. This was further increased with a disproportionate flow toward the cathode, with $12.8 \%$ for Case 3 and $12.3 \%$ for Case 4 .

\subsection{Effect of step feed on maximal power density}

Polarization tests were also performed on the unbuffered MFCs under the different operating conditions. The maximum power density without using step-feed operation was $3.5 \mathrm{~W} \mathrm{~m} \mathrm{~m}^{-3}$

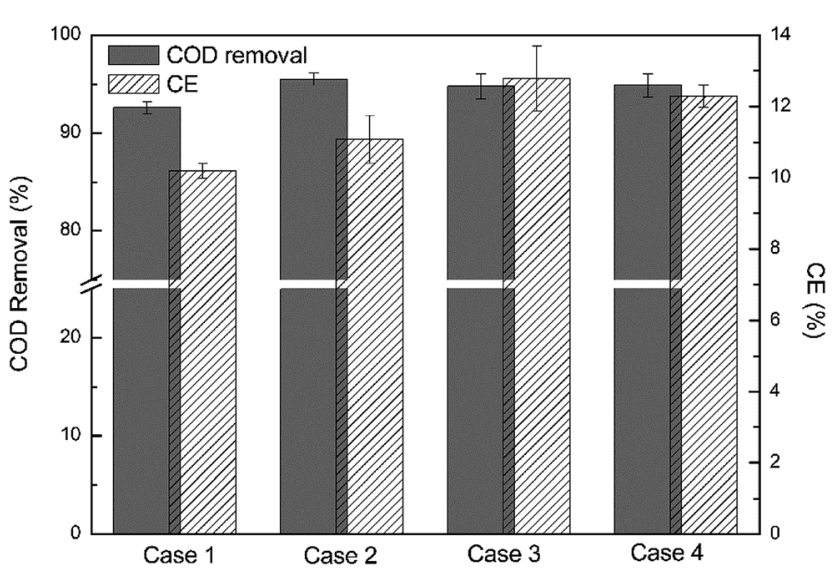

Fig. 3 COD removal and Coulombic efficiency in batch operation of unbuffered MFC under different cases.
(Fig. 4a). This improved to $4.12 \mathrm{~W} \mathrm{~m}^{-3}$ after switching to uniform step-feed operation under Case 2. It was observed that the cathode potential significantly improved, while the anode potential increased only slightly (Fig. 4b). This was probably attributed to the benefits of low $\mathrm{pH}$ adjacent to the surface of the cathode as shown in batch operation (Fig. 2c). The relatively low $\mathrm{pH}$ for the cathode reaction was attributed the enhanced proton transfer from the step-feed operation. A further 5.1\% increase in the maximum power density $\left(4.33 \mathrm{~W} \mathrm{~m}^{-3}\right)$ was found when operated under Cases 3 and 4, indicating that uneven flow distribution rates in step-feed operation could offer further improvements in unbuffered MFCs. It was reported that nonuniform current distribution was observed on large anode electrodes due to uneven ohmic resistance distribution resulting from the different electrode spacing. ${ }^{22}$ The further the anode segment was away from the cathode, the smaller the segment current generation contributed to the total current. ${ }^{22}$ It was expected that, in the present study, the upper anode brush had higher current generation and more proton production. As a result, a further performance improvement was obtained by compensating more alkaline catholyte toward the anode section closest to the cathode in step-feed of anolyte recirculation.

\subsection{Implication of the results and outlook}

Future practical application of large-scale MFCs will require strategies for operating under buffer-less conditions due to cost and environmental considerations. The previous study compared the MFC operation under buffer and unbuffered conditions to evaluate whether anolyte recirculation could replace phosphate buffer addition in air-cathode microbial fuel cells. ${ }^{\mathbf{1 4}}$ The results showed that, compared with buffer condition, MFC operated under buffer-less condition had a $27 \%$ lower voltage and $9.7 \%$ lower power density but a $64 \%$ higher Coulombic efficiency. ${ }^{\mathbf{1 4}}$ Despite this, the advantages of the buffer-less operation outweigh its drawbacks. Thus, anolyte recirculation as a method for the enhancement of proton transfer showed a potential ecofriendly and low-cost strategy for future MFC applications without adding phosphate buffer.

In this study, step-feed in anolyte recirculation was proposed to further enhance proton transfer under buffer-less condition. The results show that a step-feed configuration with uniform flow distribution across four anode sections induces better proton transfer, with a $17.7 \%$ increase in the maximum power density and a slight increase in coulombic efficiency (CE). Additional increases in power and $\mathrm{CE}$ are obtained by increasing the flow distribution toward the anode section closest to the cathode. This demonstrates that step-feed could be a beneficial operational strategy for future MFC designs. Besides the benefits of using the anolyte recirculation instead of buffers, step feed strategy has another three major advantages. Most importantly, the step-feed induces an even mass distribution not only for substrate but also for proton, resulting in a high substrate utilization and a favorable microenvironments for the growth of electrochemically active bacteria. As a result, a high COD removal and a high electrochemical activity of bacterial is obtained. In addition, for a long-term operation, it is 

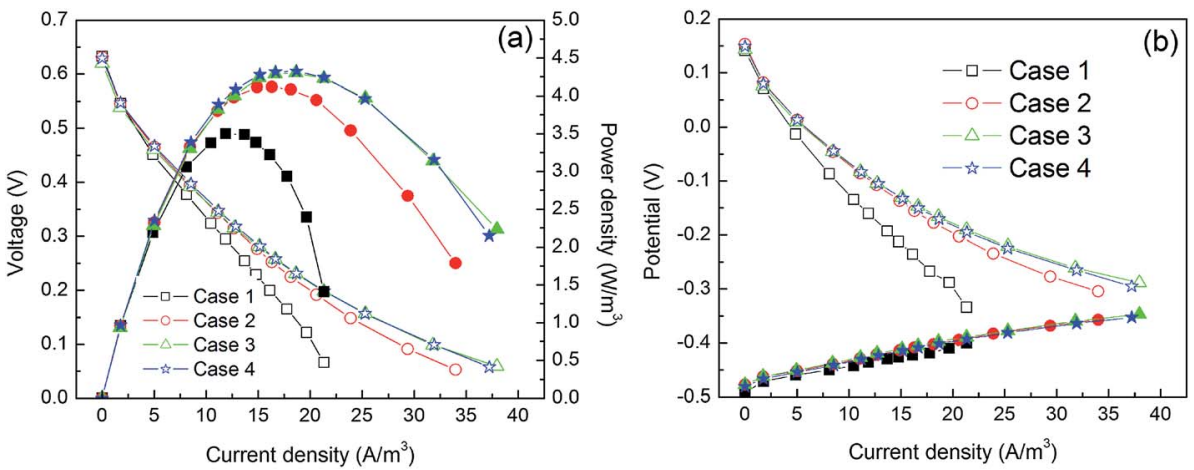

Fig. 4 Voltage and power density (a) and electrode potentials (b) during polarization testing of MFC operated under different cases (in (b), open and closed symbols indicate the cathode potential and the anode potential, respectively).

expected that this approach would lead to a more homogeneous biomass distribution along the anode electrode due to the similar microenvironments for bacterial growth. Another benefit is that a higher $\mathrm{CE}$ and power output is obtained. Conversely, additional energy required for pumping is a major operational disadvantages to operating with step-feed. In future scale-up application, the increased energy consumption from the step-feed is one of the most important considerations. In that case, the increased power from the improved individual MFC and the stacked MFCs could be helpful to compensate the additional energy consumption of pumps to a certain extent. Obviously, step-feed could be a beneficial operational strategy for future MFC designs due to the better mass transfer and more attentions should be paid on the step-feed strategy for future application of MFCs.

It is expected that step-feed strategy under buffer condition would have a higher power generation and it is unnecessary to make a buffered operation for step-feed strategy due to cost and environmental considerations. It should be noted that an unbuffered medium with a relatively lower conductivity would further deteriorate the mass transfer especially proton transfer. Thus, in MFC operated with real wastewater, step-feed is more important for the proton transfer of the unbuffered MFFCs. In addition, for future scale-up, the effects of step-feed is more significant. Our further research would be focused on the stepfeed effects in a large-scale MFCs operated with real wastewaters. The biofilm distribution on the anode electrode and microbial communities are also necessary to be investigated for better understanding of step-feed effects.

\section{Conclusions}

In this study, step-feed strategy was introduced to enhance proton transfer in unbuffered batch-fed MFCs. Compared with single-inlet feed, the introduction of step-feed induced a higher electricity generation. This was attributed to the better proton transfer induced by using step-feed in anolyte recirculation. The maximal power density and CE were improved by $17.7 \%$ and 9.9\% using step-feed operation. Further increases in power density and CE were found by increasing the recirculation rate to the anode part closest to the cathode electrode, which may have been due to nonuniform current density across the anode. These results indicate that step-feed recirculation, including uneven flow distribution, could be helpful for MFC operation. This approach could be expanded to analyze step-feed operation in continuous-flow systems.

\section{Acknowledgements}

This work was supported by the National Science Foundation for Young Scientists of China (No. 51606022), the National Natural Science Funds for Distinguished Young Scholar (No. 51325602), the National Natural Science Funds for Outstanding Young Scholar (No. 51622602), the Fundamental Research Funds for the Central Universities (106112016CDJXY145504), and the U.S. Army Research Office (W911NF-11-1-0531).

\section{References}

1 B. Logan, B. Hamelers, R. Rozendal, U. Schröder, J. Keller, S. Freguia, P. Aelterman, W. Verstraete and K. Rabaey, Environ. Sci. Technol., 2006, 40, 5181-5192.

2 W. Li and G. P. Sheng, Adv. Biochem. Eng./Biotechnol., 2012, 128, 165-197.

3 Q. Jiang, D. Xing, R. Sun, L. Zhang, Y. Feng and N. Ren, RSC Adv. , 2016, 6, 85149-85155.

4 S. Kalathil and D. Pant, RSC Adv., 2016, 6, 30582-30597.

5 W. Yang, J. Li, D. D. Ye, X. Zhu and Q. Liao, Electrochim. Acta, 2017, 224, 585-592.

6 H. Chang, Q. Fu, Y. Huang, A. Xia, Q. Liao and X. Zhu, Int. J. Agric. Biol. Eng., 2017, 10, 97-106.

7 L. Zhang, X. Zhu, J. Li, Q. Liao and D. D. Ye, J. Power Sources, 2011, 196, 6029-6035.

8 X. Shi and T. Huang, RSC Adv., 2015, 5, 102555-102559.

9 J. Li, H. J. Li, J. L. Zheng, L. Zhang, Q. Fu, X. Zhu and Q. Liao, Bioresour. Technol., 2017, 233, 1-6.

10 B. Logan, Appl. Microbiol. Biotechnol., 2010, 85, 1665-1671.

11 Z. Ge, L. Wu, F. Zhang and Z. He, J. Power Sources, 2015, 297, 260-264.

12 Y. Z. Fan, H. Q. Hu and H. Liu, Environ. Sci. Technol., 2007, 41, 8154-8158.

13 Y. Ahn and B. Logan, Bioresour. Technol., 2013, 132, 436-439. 
14 L. Zhang, X. Zhu, H. Kashima, J. Li, D. D. Ye, Q. Liao and J. M. Regan, Bioresour. Technol., 2015, 179, 26-34.

15 S. Freguia, K. Rabaey, Z. Yuan and J. Keller, Water Res., 2008, 42, 1387-1396.

16 G. C. Gil, I. S. Chang, B. H. Kim, M. Kim, J. K. Jang, H. S. Park and H. J. Kim, Biosens. Bioelectron., 2003, 18, 327-334.

17 L. Zhang, J. Li, X. Zhu, D. D. Ye and Q. Liao, Int. J. Hydrogen Energy, 2015, 40, 3953-3960.

18 F. Zhang, K. Jacobson, P. Torres and Z. He, Energy Environ. Sci., 2010, 3, 1347-1352.

19 P. Clauwaert, S. Mulenga, P. Aelterman and W. Verstraete, Appl. Microbiol. Biotechnol., 2009, 83, 241-247.

20 H. Liu, S. Cheng, L. Huang and B. Logan, J. Power Sources, 2008, 179, 274-279.
21 T. Ewing, P. Ha, J. Babauta, N. Tang, D. Heo and H. Beyenal, J. Power Sources, 2014, 272, 311-319.

22 L. Zhang, J. Li, X. Zhu, D. D. Ye and Q. Liao, Chem. Eng. J., 2013, 223, 623-631.

23 Z. Wang, M. Huang, R. Qi, S. Fan, Y. Wang and T. Fan, Chem. Eng. J., 2017, 314, 291-300.

24 G. Cao, S. Wang, Y. Peng and Z. Miao, Bioresour. Technol., 2013, 128, 604-611.

25 G. Zhu, Y. Peng, B. Ma, Y. Wang and C. Yin, Chem. Eng. J., 2009, 314, 195-201.

26 S. Yang, M. Gao, S. Liang, S. Wang and X. Wang, Bioresour. Technol., 2013, 143, 682-685. 